\title{
The rotation curve and the density model of the Milky Way
}

\author{
Oleksiy Golubov and Andreas Just \\ Astronomisches Rechen-Institut, Zentrum für Astronomie der Universität Heidelberg, \\ Mönchhofstraße 12-14, 69120 Heidelberg, Germany, \\ email: golubov@ari.uni-heidelberg.de
}

\begin{abstract}
We study the asymmetric drift in the Milky Way with the aid of the RAVE data. Then we apply the deduced asymmetric drift correction to the SEGUE data and reconstruct the behaviour of the rotation curve of the Milky Way in the extended solar neighbourhood. The rotation curve appears to be essentially flat. We supplement our data by tangent point measurements of the inner rotation curve and fit it by a density model of the Milky Way.
\end{abstract}

Keywords. Galaxy: kinematics and dynamics, Galaxy: solar neighborhood

We study the asymmetric drift of the Milky Way disc dwarf stars with the sample from the Radial Velocity Experiment (RAVE). We adopt photometric parallaxes by Zwitter et al. (2010), bin the stars in colour, and study the mean rotational velocity as function of the squared radial velocity dispersion. We find that the linear Strömberg's relation provides a good fit to the data, with the best fit parameters $R_{d}=2.37 \mathrm{kpc}$ for the radial scale length of the disc and $V_{\odot}=4.37 \mathrm{~km} \mathrm{~s}^{-1}$ for the $V$-component of the velocity of the Sun with respect to the local standard of rest. When the RAVE data are divided into 2 bins in the Galactic radius and the asymmetric drift correction is performed, we get 2 points in the rotation curve, nearly $300 \mathrm{pc}$ inside and outside the solar radius. There is only a slight hint on the decrease of the circular velocity in the solar neighbourhood.

Then we apply the same Strömberg's relation to the F- and G-dwarfs sample from SDSS Extension for Galactic Understanding and Exploration (SEGUE). Photometric parallaxes of the stars were measured by Lee et al. (2011). The stars are at distances up to about $2 \mathrm{kpc}$ from the Sun, and the deduced rotation curve is almost flat over the range of Galactocentric radii 7 to $10 \mathrm{kpc}$, in contrast to Sofue et al. (2009), who assume a strong dip in the rotation curve just outside the solar radius.

We supplement our data for the local rotation curve with the tangent point data for the inner rotation curve from Sofue et al. (2009), and fit them by a theoretical rotation curve, corresponding to a 3-component density model of the Galaxy, consisting of a Dehnen bulge, an exponential disc with a hole, and a flattened dark matter halo (Golubov \& Just 2013). During this fitting we keep the local surface density and dark matter density in the disc constrained to the values determined by Just \& Jahreiß (2010). Thus the density model of the Milky Way is constructed.

\section{References}

Golubov, O. \& Just, A. 2013, in: T. Wong \& J. Ott (eds.), Molecular Gas, Dust, and Star Formation in Galaxies, Proceedings of IAU Symposium No. 292 (Cambridge: Cambridge University Press)

Just, A. \& Jahreiß, H. 2010, MNRAS, 402, 461

Lee, Y. S., Beers, T. C., An, D., Ivesic, Z., Just, A. et al. 2011, ApJ, 738, 187

Sofue, Y., Honma, M., \& Omodaka, T. 2009, PASJ, 61, 227

Zwitter, T., Matijevič, G., Breddels, M. A., et al. 2010, A\&̊A, 522, 54 